\title{
Can a photonic thermalization gap arise in disordered non- Hermitian Hamiltonian systems?
}

\author{
Guang-Lei Wang ${ }^{1}$, Hong-Ya Xu ${ }^{1}$, Ying-Cheng LaI ${ }^{1,2}$ \\ 1 School of Electrical, Computer and Energy Engineering, Arizona State University, Tempe, Arizona 85287, USA \\ 3 Department of Physics, Arizona State University, Tempe, Arizona 85287, USA
}

PACS 05.45.-a - Nonlinear dynamics and chaos

PACS 05.45.Mt - Quantum chaos; semiclassical methods

PACS 42.82.Et - Waveguides, couplers, and arrays

\begin{abstract}
The phenomenon of chiral-symmetry protected thermalization gap in Hermitian photonic systems is counterintuitive as it implies that the photon coherence can be continuously improved by disorders towards an asymptotic limit. We show that the phenomenon disappears in time-independent, non-Hermitian photonic systems even when the chiral symmetry is well preserved. In fact, the degree of thermalization generally increases with the disorder strength, in agreement with intuition. As non-Hermitian characteristics (e.g., weak gain and loss) can be expected in realistic physical situations, the phenomenon of thermalization gap may be observed but only in well controlled experiments with high quality materials.
\end{abstract}

Introduction. Recent years have witnessed a rapid growth of interest in non-Hermitian systems $[1,2$. In open Hamiltonian systems with symmetry breaking so that the Hermitian properties can no longer be maintained, surprising physical phenomena can arise 38 . For example, in condensed matter physics, the principle of bulk-boundary correspondence stipulates that the bulk of the lattice can be characterized by a topological invariant whose value determines the existence of possible gapless edge states. However, a recent work 9 demonstrated that violating the Hermitian properties can induce a fractional topological invariant number and lead to a change in the stability of the edge states, thereby defying the bulk-boundary correspondence. Another example occurs in wave propagation/transport, where conventional wisdom is that phenomena such as diffusion and localization are caused by random disorders. However, it was demonstrated theoretically and experimentally 10,11 that a sudden transition from ballistic to diffusive motions can occur even in ordered, time-independent, non-Hermitian systems maintaining the parity-time reversal $(\mathcal{P} \mathcal{T})$ symmetry. Also, a remarkable phenomenon in non-Hermitian systems is the appearance of exception points in the eigenvalue spectrum about which crossing/anti-crossing transitions can occur and the corresponding emergence of a new topological Berry phase associated with paths encircling the exceptional points 12,13 . In physical contexts such as resonances and discrete-continuous energy transitions, the eigenvalues of the Hamiltonian are necessarily complex to account for, e.g., the finite lifetime of the state. Similarly, in non-Hermitian systems, the eigenvalues are complex. Experimentally, non-Hermitian physics can be implemented and observed in waveguides, photonic crystals, dielectric micro-resonators, and even in biological neutral networks 14].

In one-dimensional Hermitian systems, the emergence of a chiral symmetry protected photonic thermalization gap has been theoretically predicted 15,17$]$ and observed experimentally 17, 19]. A system is said to possess a chiral symmetry if (a) there are eigenvalues (labeled by integers $m$ and $-m$ ) that appear in pairs whose real and imaginary parts have opposite signs, and (b) the associated eigenstates satisfy the relation: $\phi_{n}^{m}=(-1)^{n} \phi_{n}^{-m}$, where $n$ is the space coordinate. Of interest is how random disorders affect the statistical properties of photon thermal fluctuations. To measure the statistical properties of these fluctuations, a second-order coherence function, i.e., the normalized intensity correlation $g^{(2)}$ [Eq. 7 below], is commonly used 15, 17, 20], which is capable of revealing statistical information about the source. The values of $g^{(2)}$ are one for fully coherent light (e.g., laser) and two for random or chaotic light (e.g., black body radiation). In the absence of any disorder, there is a high degree of coherence among the photons. In this case, $g^{(2)}$ 
assumes the unity value corresponding to coherent photon states. When there are arbitrarily weak disorders, i.e., when the strength of disorder is turned on from zero, there is an abrupt transition in $g^{(2)}$ from unity to a value corresponding to incoherence at which the photon statistics can be approximately described by the modified Bose-Einstein distribution. As the disorder strength is further increased, the value of $g^{(2)}$ decreases but can never reach the unity value again in systems with a chiral symmetry. In fact, the lower bound of the measure has the value of two at which the Bose-Einstein distribution holds. A "thermalization gap" thus arises in the plot of the measure versus the disorder strength 15,17 .

The emergence of a photon thermalization gap $15-19$ is striking and counterintuitive, as it implies that, beyond the regime of weak disorder, the degree of photon coherence in the underlying system can be continuously improved by increasing the disorder strength. This result was obtained for purely Hermitian systems with a chiral symmetry, e.g., a photonic system of a set of ideal parallel coupled waveguides with the values of the refractive index being purely real, where there is no emission and/or absorption. In physical reality the refractive index of any material can be expected to be generically complex with an inevitable nonzero imaginary part. This consideration motivated us to investigate thermalization in non-Hermitian photonic systems.

To choose a prototype system setup, we note that, in principle, we can impose certain symmetries on the nonHermitian system. However, special arrangement of the configuration may be required. For example, if we demand that the system be time independent and possess a $\mathcal{P} \mathcal{T}$ symmetry, the real and imaginary parts of the refractive index must be an even and odd function in space, respectively 21,22]. Although $\mathcal{P} \mathcal{T}$ symmetry without such a special arrangement can be realized, time modulation or spatial engineering of the material along the propagation direction is required 23 . We are thus led to consider the more general setting where we assume that the non-Hermitian system is time independent and does not possess a $\mathcal{P} \mathcal{T}$ symmetry. Specifically, we study the photon thermalization statistics in photonic systems of waveguide array in which the refractive index of each individual element can be complex with either sign (i.e., gain or loss). To be more general, we consider disorders that can either retain or break the chiral symmetry. We develop a framework of coupled mode equations for non-Hermitian waveguide systems with varying separations and refractive indices, which enables us to address the question of whether a chiral-symmetry protected thermalization gap can arise in non-Hermitian photonic systems. The main finding is that the degree of photon coherence continues to deteriorate as the disorder strength is increased, a result in sharp contrast to that in Refs. 15-17]. Our work thus establishes the necessary conditions under which a thermalization gap can be expected in a photonic system. In particular, unless in a well controlled experiment with nearly perfect materials in the intermediate time (propagation distance) regime, the counterintuitive phenomenon that disorder can continue to improve the coherence of photon fluctuations cannot occur. In fact, in non-Hermitian systems no thermalization gap can be expected.

Non-Hermitian photonic systems. Hermitian symmetry requires $H=H^{\dagger}$ and the system is chiral symmetric if $C H C=-H$, with $C=\operatorname{diag}(1,1, \ldots, 1,-1, \ldots,-1)$ being the chiral symmetry operator. In general, the Hamiltonian matrix of a photonic waveguide system can be expressed in a $2 \times 2$ block form:

$$
H \rightarrow\left[\begin{array}{cc}
H^{\prime} & H_{S} \\
H_{S}^{T} & H^{\prime \prime}
\end{array}\right]
$$

where $H^{\prime}, H^{\prime \prime}$ and $H_{S}$ are matrices in the corresponding blocks. For a system with chiral symmetry, the diagonal blocks are zero: $H^{\prime}=H^{\prime \prime}=0$. Further, if the system is Hermitian, the left-bottom block can be expressed as $H_{S}^{\dagger}$. In this case, the Hamiltonian is real, which is the class of systems in which the phenomenon of a thermalization gap $15-19$ was uncovered. For systems considered in our work, we have $H_{S}^{T} \neq H_{S}^{\dagger}$, and the Hamiltonian is in general complex.

As a concrete setting to investigate the photon statistics in non-Hermitian systems, we consider an array of onedimensional, single-mode optical waveguides arranged in the $x$ direction with gain and loss, as shown in Fig. 1. Each waveguide has complex relative permittivity $\epsilon_{n}+i \epsilon_{n}^{\prime}$, and the waveguides are placed in a surrounding medium with complex permittivity $\epsilon_{0}+i \epsilon_{0}^{\prime}$. In the idealized situation where the waveguides are identical and equally spaced, the system can be described by a set of complex, coupled mode equations 11 . In a realistic situation, random factors are present, which introduce disorders that can be modeled as proper perturbations to the coupled mode equations.

We start from the Helmholtz equation governing wave propagation in the system:

$$
\left[\nabla^{2}+k_{0}^{2} \tilde{\epsilon}(x)\right] \Psi(x, z)=0,
$$

where $z$ is the propagation direction, $\Psi(x, z)$ is the electric field amplitude, $k_{0}$ is the free space wave vector, and $\tilde{\epsilon}(x)$ is the profile of the relative electric permittivity of the system, which can be expressed as

$$
\tilde{\epsilon}(x)=\epsilon_{0}+i \epsilon_{0}^{\prime}+\sum_{n=1}^{N}\left[\left(\epsilon_{n}-\epsilon_{0}\right)+i\left(\epsilon_{n}^{\prime}-\epsilon_{0}^{\prime}\right)\right] \zeta_{n}(x),
$$

where $2 w$ is the width of the each waveguide and $\zeta_{n}(x)=$ $\Theta\left(x-x_{n}+w\right)-\Theta\left(x-x_{n}-w\right)$ with $\Theta(x)$ being the Heaviside step function. The effective coupled mode equations can be obtained under the approximation that the eigenmode of the individual waveguide is well confined so that the full width half maximum (FWHM) $\ell$ is much smaller than $d$, the spacing between two adjacent waveguides. The equations are 11

$$
-i \frac{d \phi_{n}}{d z}=i \kappa \phi_{n}+\left(C+i C^{\prime}\right)\left(\phi_{n+1}+\phi_{n-1}\right)
$$




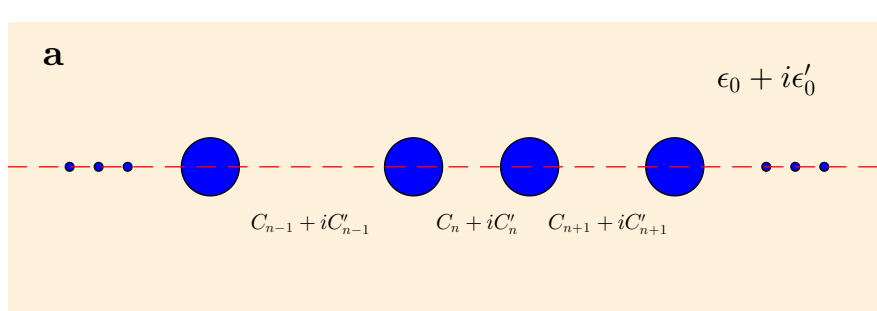

b

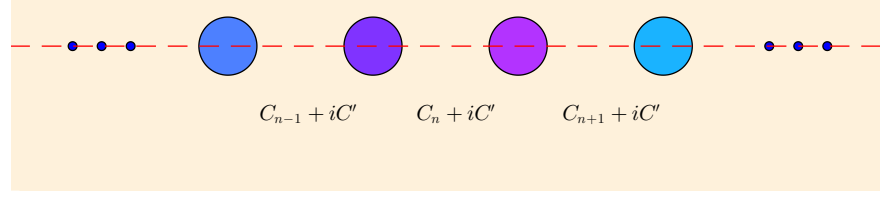

Fig. 1: Two types of disorders in a non-Hermitian photonic system of a waveguide array. Both the waveguides and the surrounding medium have a complex reflection index. (a) Spacing disorder in which the distances between the nearest waveguides are randomly modified. The disorders will result in randomness in both the real and imaginary parts of the coupling constant. (b) Relative permittivity disorder in which the complex refractive index of each waveguide is randomly modified while the distances between the nearest waveguides are kept constant. As a result, there will be random perturbations upon both the diagonal and off-diagonal elements of the Hamiltonian.

where

$$
\begin{aligned}
\kappa & =\frac{k_{0}^{2}}{2 \beta}\left[\epsilon_{0}^{\prime}+\left(\epsilon^{\prime}-\epsilon_{0}^{\prime}\right) \tanh \left(\frac{w}{\ell}\right)\right], \\
C & =\frac{\left(\epsilon-\epsilon_{0}\right) k_{0}^{2}}{2 \beta} \frac{w}{\ell} \exp \left(-\frac{d}{\ell}\right), \\
C^{\prime} & =\frac{\left(\epsilon_{0}^{\prime}-\epsilon^{\prime}\right) k_{0}^{2}}{\beta} \frac{d}{\ell} \exp \left(-\frac{d}{\ell}\right) .
\end{aligned}
$$

The quantity $\phi_{n}$ in Eq. (4) is the field amplitude in the $n$th waveguide and $k_{0} \sqrt{\epsilon_{0}}<\beta<k_{0} \sqrt{\epsilon}$ is the propagation constant along the $z$ direction.

To incorporate disorder-immune chiral symmetry in the non-Hermitian waveguide system, we consider two types of disorders. For the first type, the distance between a pair of adjacent waveguides is randomly modified, as shown in Fig. 1(a). Mathematically, insofar as the approximation $\ell \ll\left|x_{n}-x_{n-1}\right|$ holds, the general form of the coupled mode equations remains the same, except that the coupling constant now has a spatial dependence:

$$
\begin{aligned}
C \rightarrow C_{n, n \pm 1}= & \frac{\left(\epsilon-\epsilon_{0}\right) k_{0}^{2}}{2 \beta} \frac{w}{\ell} \exp \left(-\frac{\left|x_{n}-x_{n \pm 1}\right|}{\ell}\right) \\
C^{\prime} \rightarrow C_{n, n \pm 1}^{\prime}= & \frac{\left(\epsilon_{0}^{\prime}-\epsilon^{\prime}\right) k_{0}^{2}}{\beta} \frac{\left|x_{n}-x_{n \pm 1}\right|}{\ell} \\
& \exp \left(-\frac{\left|x_{n}-x_{n \pm 1}\right|}{\ell}\right)
\end{aligned}
$$

where $\left|x_{n}-x_{n \pm 1}\right|=d\left(1+\xi_{1}\right)$ and $\xi_{1}$ is a random variable uniformly distributed in $[-\Delta C, \Delta C]$. We consider the weak disorder regime and retain the first order corrections. In general, random modulation in the waveguide spacing leads to disorders in both the real and imaginary parts of the coupling constant. In a matrix representation of the coupled mode equation, this type of disorders will be present in the off-diagonal elements only, hence the term "off-diagonal disorders." In spite of the presence of waveguide spacing disorders, the system is still chiralsymmetric.

While the off-diagonal disorders described above preserve the chiral symmetry of the non-Hermitian system, the second type of disorders we study break the chiral symmetry. Conventionally, this can be done by introducing disorders into the diagonal elements of the coupling matrix. However, as can be seen from Eq. (5), it is not feasible to introduce diagonal disorders while keeping the off-diagonal elements unperturbed. Our strategy is then to randomly modify the real part of the relative permittivity of the individual waveguides while keeping the imaginary part identical. This way both diagonal and off-diagonal disorders are present to break the chiral symmetry, as shown in Fig. 1(b). Mathematically, including this kind of disorders entails altering the quantities $\kappa$ and $C$ in the following manner:

$$
\begin{aligned}
& i \kappa \rightarrow \quad i \kappa+\frac{\left(\epsilon_{n}-\bar{\epsilon}\right) k_{0}^{2}}{2 \beta} \tanh \left(\frac{w}{\ell}\right), \\
& C \rightarrow \quad C_{n, n \pm 1}=\frac{\left(\epsilon_{n}+\epsilon_{n \pm 1}-\bar{\epsilon}-\epsilon_{0}\right) k_{0}^{2}}{2 \beta} \frac{w}{\ell} \exp \left(-\frac{d}{\ell}\right),
\end{aligned}
$$

where $\epsilon_{n}$ is the real relative permittivity of the $n$th waveguide and $\bar{\epsilon}$ is the corresponding average value of all the waveguides. We set $\epsilon_{n}=\epsilon\left(1+\xi_{2}\right)$, where $\xi_{2}$ is a random variable uniformly distributed in $[-\Delta \beta, \Delta \beta]$.

To characterize the photon statistics in the nonHermitian system, we use the following three quantities 15]: the normalized intensity correlation $g^{(2)}(z)$, the intensity probability distribution $P(\bar{\mu})$, and the photonnumber distribution $P\left(n_{p h}\right)$. In particular, $g^{(2)}(z)$ characterizes the degree of randomness of light in the probability space of disorder realizations, which is defined as 15

$$
g_{x}^{(2)}(z)=\frac{\left\langle I_{x}^{2}(z)\right\rangle}{\left\langle I_{x}(z)\right\rangle^{2}}
$$

where $I_{x}(z)=\left|\phi_{x}(z)\right|^{2}$ is the intensity of the electric field at the $x$ th waveguide and $\langle\cdot\rangle$ denotes ensemble averaging. For coherent light, we have $g^{(2)}=1$. For thermalized (random or chaotic) light, we have $g^{(2)}=2$, where the intensity correlation can be evaluated at the middle of the waveguide array 15,16$]$. One may also consider the normalized intensity correlation between a pair of waveguides, from which non-Gaussian statistics corresponding to photon antibunching have recently been found [24].

The intensity probability distribution $P(\bar{\mu})$ and the full photon-number distribution $P\left(n_{p h}\right)$ can exhibit different scaling behaviors for different degrees of photon thermalization, e.g., coherent, thermalized/chaotic and superchaotic. In our study, we set the initial excitation at the 
center of the waveguide array and generate a large number of realizations of the input intensity, which obey the coherent Poisson distribution. After the excitation propagates in the waveguides for certain distance (which is effectively time), we examine the intensity at the central waveguide, which is proportional to the photon numbers. The photon-number distribution can be obtained through the ensemble statistics after considering the effect of measurement.

For a Hermitian system, the conventional solution approach is to expand the initial wavefunction in terms of the eigenfunctions and then to calculate the time evolution of each eigenfunction. However, this approach is not suitable for non-Hermitian systems, as the eigenfunctions of a non-Hermitian matrix are generally not orthogonal to each other. We thus resort to direct numerical solutions of the coupled mode equations. For convenience, we use the same parameters as in the experimental work [11: $C_{0}=0.1 \mathrm{~mm}^{-1}$ to rescale all the parameters, and set

$$
\alpha=2 \frac{\left(\epsilon_{0}^{\prime}-\epsilon^{\prime}\right)}{\left(\epsilon-\epsilon_{0}\right)} \frac{d}{w}=-0.15
$$

to relate $C^{\prime}$ to $C_{0}$ and $\kappa / C_{0}=0.3$. For adjacent distance disorders, since the unaffected diagonal elements can be removed by incorporating the quantity $e^{-\kappa t}$ into all the wave amplitudes, i.e., $\phi(z)=E(z) e^{-\kappa t}$. This extra time evolution term contributes to an overall gain or loss, depending on the sign of $\kappa$, but it is identical for all disorder realizations, which can be canceled out when calculating $g_{0}^{(2)}$. For disorders in the real relative permittivity, since the imaginary part is constant, the diagonal $i \kappa$ term can still be removed. Other parameters are set to be $d=17 \mu \mathrm{m}$, $2 w=5 \mu \mathrm{m}, \epsilon_{0} / \epsilon=0.2$, and $d / \ell \sim 2$.

We choose the initial condition as a single excitation at the central waveguide. For a Hermitian system, this choice guarantees that both modes in each chiral pair have the same weight during propagation, activating the chiral symmetry. However, in a non-Hermitian system, orthogonality and completeness are not guaranteed for the eigenvectors. While it may be possible to design an input excitation for any specific disorder realization so that the symmetric-excitation condition is fulfilled, the gain/loss effects would induce imbalances between each pair of modes during propagation, defying the symmetricexcitation condition.

Main results. To verify the chiral symmetry in our nonHermitian system, we examine the eigenvalue spectrum and the associated eigenfunctions for a typical realization of the off-diagonal disorder, as shown in Fig. 2. Mathematically, the chiral symmetry requirement $C H C=-H$ leads to $\xi_{m}=-\xi_{-m}$ and $\phi_{n}^{m}=(-1)^{n} \phi_{n}^{-m}$, where $\xi_{m}$ stands for the real or imaginary part of the $m$ th eigenvalue and $\phi_{n}^{m}$ is the corresponding eigenfunction at the $n$th waveguide. Figure 2 reveals a continuous existence of the chiral symmetry even in the presence of off-diagonal, complex disorders. An alternative way to test the chiral
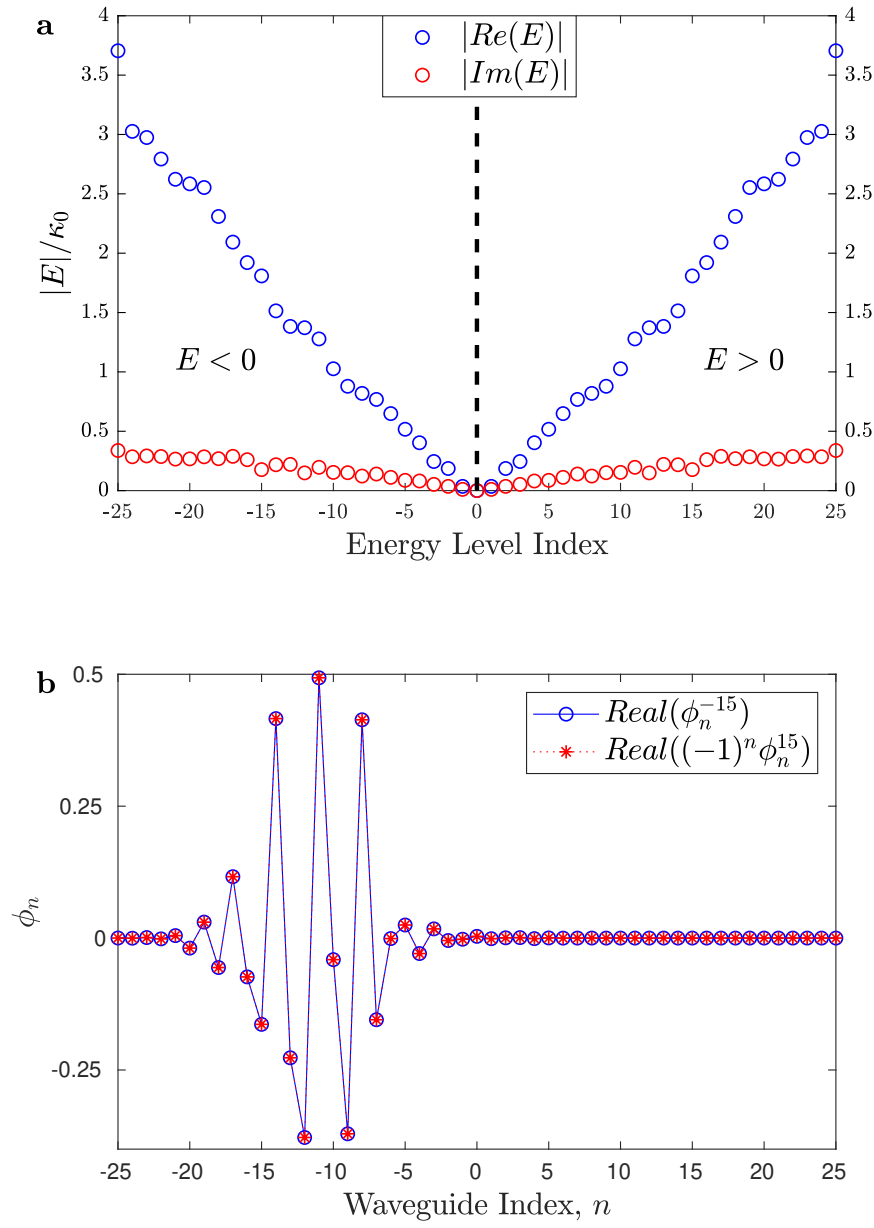

Fig. 2: Properties of non-Hermitian systems with offdiagonal disorder. (a) Complex eigenvalue spectrum exhibiting an odd symmetry for both the real and imaginary parts: $\xi_{m}=-\xi_{-m}$. It is convenient to take the absolute values of the eigenvalues. The energies in the left panel are negative. (b) A representative pair of eigenfunctions with the property $\phi_{n}^{m}=(-1)^{n} \phi_{n}^{-m}$. Shown are the real parts of the eigenfunctions with $m=-15$. The imaginary parts share the same symmetry.

symmetry is to calculate the commutation between the Hamiltonian and the chiral symmetry operator. We note that, if the disorders occur in the real relative permittivity, the chiral symmetry will be broken.

Figure 3 summarizes our main finding that in nonHermitian systems, the previously reported chiral symmetry protected photonic thermalization gap disappears. In particular, Figs. 3(a,b) show the dependence of the normalized intensity correlation $g_{0}^{(2)}$ on the propagation distance and the disorder strength for the two types of disorders. In both cases, a long propagation distance and a high disorder strength can lead to a relatively high value $g_{0}^{(2)}$. This can be understood by noting that the main source of the randomness in the intensity is the gain/loss that behaves as $\sim \exp \left(\Delta\left[\operatorname{Im}\left(E_{n}\right)\right] \cdot t\right)$. A higher level of disorder thus leads to a larger value of $\Delta\left[\operatorname{Im}\left(E_{n}\right)\right]$, while a 

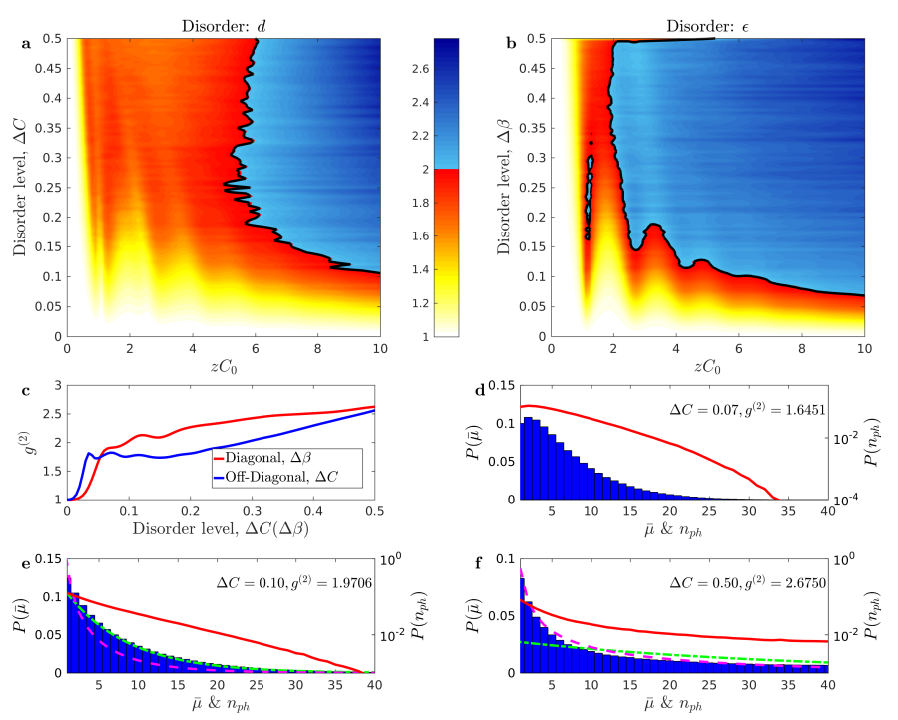

Fig. 3: Behavior of photon thermalization in nonHermitian systems subject to random disorders. (a,b) Normalized intensity correlation $g_{0}^{(2)}(z)$ at the center of the waveguide array as a function of the disorder strength and propagation distance $z$ for a system with adjacent distance disorder (off-diagonal disorder) and real relative permittivity disorder, respectively. The number of waveguides in one array is 51. (c) The correlation measure $g_{0}^{(2)}\left(z C_{0}=10\right)$ as a function of the disorder strength for off-diagonal (blue) and permittivity (red) disorders. (d-f) Intensity probability distribution $P(\bar{\mu})$ and photon number distribution $P\left(n_{p h}\right)$ for a coherent input with a fixed mean photon number $\bar{\mu}=680$ at the input for different values of the off-diagonal disorder strength. The intensity $I=|\phi|^{2}$ is represented by the mean photon number $\bar{\mu}$ while the initial photon number is set to be $I=1$. The left axis indicates $P(\bar{\mu})$ on a linear scale (the blue bar) while the right axis is a semi-logarithmic representation of $P\left(n_{p h}\right)$ (red). The dashed-dot and the dashed curves represent the fitted exponential and Gaussian-square distributions, respectively, in which the disorder strengths and the corresponding $g^{(2)}$ values are inserted. Due to the system's being non-Hermitian, eigenvalues are complex. Numerically it is necessary to set a finite (albeit relatively large) distance to prevent energy from diverging.

longer propagation distance corresponds to a longer time. For the second type of disorders (i.e., real relative permittivity disorders), the values of $g_{0}^{(2)}$ are generally larger as compared to those with the first type of disorders (i.e., adjacent distance or off-diagonal disorders), due to the fact that a nonzero value of $\Delta \beta$ induces both diagonal and offdiagonal disorders. We observe that, in the small time regime, the main signatures of the Hermitian characteristics of the system as reported in Ref. [15] are retained, i.e., $g_{0}^{(2)}$ tends to decrease with the disorder strength. However, in the long time (or equivalently, large distance) regime, $g_{0}^{(2)}$ increases with the disorder strength. In this regime, the non-Hermitian characteristics dominate, as the main contributions of the imaginary parts of the eigenvalues are from the exponential term. As a result, an in- crease in the disorder strength will be amplified by the propagation distance, leading to a continuous increase in the value of $g_{0}^{(2)}$.

Figure 3(c) shows the behavior of $g_{0}^{(2)}$ versus the disorder strength for $z C_{0}=10$. We observe a non-decreasing behavior, where the value of $g_{0}^{(2)}$ changes continuously from unity to about 3. For longer propagation length, the value of $g_{0}^{(2)}$ can exceed 3 , due to the non-Hermitian nature of the system. Say we consider $R$ realizations of the off-diagonal order. Except for the overall gain/loss factor $\kappa$, the imaginary parts that govern the evolution of the eigenmodes are random. After a long time, the difference in the intensities of the eigenmodes grows exponentially, resulting in significant differences in the intensities of the central waveguide among different realizations. In such a case, we have

$$
g_{0}^{(2)}(z)=\frac{\left\langle I_{0}^{2}(z)\right\rangle}{\left\langle I_{0}(z)\right\rangle^{2}} \simeq \frac{I_{0, \max }^{2}(z) / R}{\left(I_{0, \max }(z) / R\right)^{2}}=R
$$

indicating that $g_{0}^{(2)}$ can approach the upper bound $R$.

Figures $3(\mathrm{~d}-\mathrm{f})$ show the full photon number statistics for the case of off-diagonal disorders for $z C_{0}=10.0$. From the initial condition, we generate an ensemble of input intensity, with the distribution which can approximately be described by the Poisson form: $P(n)=\bar{\mu}^{n} e^{-\bar{\mu}} / n$ ! with the mean photon number $\bar{\mu} \approx 680$. Due to gain, the effective electric field $E(z)$ can reach a large value. Since $\phi(z)=E(z) e^{-\kappa t}$, the real electric field amplitude can still be moderate for an appropriate value of $\kappa$. Note that the expression of $\kappa$ does not depend on the nature of the disorders. We can thus adjust the value of $\kappa$ to ensure that the energy of the system has some reasonable value within a finite distance. Experimentally, the range of the accessible imaginary relative permittivity can be large, where gain can be realized e.g., through photon pumping while loss can be introduced by metal.

Figure $3(\mathrm{~d})$ shows a case where $g_{0}^{(2)}=1.6451$ and there is a transition from the coherent Poisson distribution to the thermalized Gaussian distribution. About the normal thermal state for which $g_{0}^{(2)} \approx 2$, the intensity distribution is exponential: $P(I)=(1 / \mu) e^{-I / \mu}$, where $\mu=\left\langle n_{p h}\right\rangle$ is the ensemble averaged photon number and the photon number statistics follow the Bose-Einstein distribution 15. For the superthermal state $g_{0}^{(2)} \approx 3$, the intensity should follow a Gaussian-square type of distribution: $P(I)=(1 / \sqrt{\pi \mu I}) e^{-I / \mu}$, which is associated with a modified Bose-Einstein photon number statistics [15]. These behaviors have indeed been observed. In particular, in Figs. 3(e,f), the left side axis is for the blue bar and is on the linear scale of $P(\bar{\mu})$, and the right axis is for the red curve $P\left(n_{p h}\right)$ on a semi-logarithmic scale, where the mean photon number $\bar{\mu}$ is used to mark the strength of the intensity $I$. From Fig. $3(\mathrm{e})$, we have $g_{0}^{(2)} \approx 1.97$ and there is an approximately linear dependence of $P\left(n_{p h}\right)$ on $n_{p h}$ on the semi-logarithmic scale, indicating a Bose-Einstein photon 
number distribution. The dashed-dot green fitting line for the exponential intensity distribution agrees with the blue bar. In Fig. 3(f), we have $g_{0}^{(2)} \approx 2.68$, the dependence of $P\left(n_{p h}\right)$ on $n_{p h}$ on the semilogarithmic scale indicates a superthermal distribution, where the dashed magenta fitting curve obeys the Gaussian-square distribution.

Conclusions. By developing a form of the coupled mode equations for non-Hermitian disordered waveguides systems, we have addressed the question of whether the recently discovered phenomenon of chiral symmetry protected thermalization gap can arise in non-Hermitian waveguide systems. We have found that Hermitian symmetry is a necessary condition for the thermalization gap. In particular, we have studied the photon number statistics for two types of random disorder: one preserving and another breaking the chiral symmetry, with the finding that, regardless of whether there is a chiral symmetry, the system's being Hermitian is key to the emergence of a photonic thermalization gap. Non-Hermitian photonic systems with gain and loss are typical in realistic situations. For such systems the underlying photon statistics will become progressively random with the disorder level, eliminating the possibility of the emergence of any photonic thermalization gap.

Previously, non-Hermitian effects are incorporated into the coupled mode equations in a phenomenological way [11, where even identical homogeneous nonHermitian waveguides possess a complex coupling coefficient. We have gone beyond the configuration of identical homogeneous waveguides and derived a more general form of the non-Hermitian coupled mode equations. While the focus of our work is on disorder effects, our general coupled mode equations are suitable for investigating other system configurations, insofar the coupled mode and small perturbation approximations hold.

$$
* * *
$$

We would like to acknowledge support from the Vannevar Bush Faculty Fellowship program sponsored by the Basic Research Office of the Assistant Secretary of Defense for Research and Engineering and funded by the Office of Naval Research through Grant No. N00014-16-1-2828.

\section{REFERENCES}

[1] Cao H. and Wiersig J., Rev. Mod. Phys., 87 (2015) 61.

[2] Konotop V. V., Yang J. and Zezyulin D. A., Rev. Mod. Phys., 88 (2016) 035002.

[3] del Campo A., Egusquiza I. L., Plenio M. B. and Huelga S. F., Phys. Rev. Lett., 110 (2013) 050403.

[4] Regensburger A., Miri M.-A., Bersch C., Näger J., Onishchukov G., Christodoulides D. N. and Peschel U., Phys. Rev. Lett., 110 (2013) 223902.

[5] Lee T. E., Reiter F. and Moiseyev N., Phys. Rev. Lett., 113 (2014) 250401.

[6] Lee T. E. and Chan C.-K., Phys. Rev. X, 4 (2014) 041001 .
[7] Malzard S., Poli C. and Schomerus H., Phys. Rev. Lett., 115 (2015) 200402.

[8] Sarma R., Ge L., Wiersig J. and Cao H., Phys. Rev. Lett., 114 (2015) 053903.

[9] LeE T. E., Phys. Rev. Lett., 116 (2016) 133903.

[10] Eichelkraut T., Heilmann R., Weimann S., Stützer S., Dreisow F., Christodoulides D., Nolte S. and Szameit A., Nat. Commun., 4 (2013) .

[11] Golshani M., Weimann S., Jafari K., Nezhad M. K., Langari A., Bahrampour A., Eichelkraut T., MahDavi S. and Szameit A., Phys. Rev. Lett., 113 (2014) 123903.

[12] Gao T., Estrecho E., Bliokh K., Liew T., Fraser M., Brodbeck S., Kamp M., Schneider C., Höfling S., Yамамото Y. et al., Nature, 526 (2015) 554.

[13] Liertzer M., Ge L., Cerjan A., Stone A. D., Türeci H. E. and Rotter S., Phys. Rev. Lett., 108 (2012) 173901.

[14] Amir A., Hatano N. and Nelson D. R., Phys. Rev. E, 93 (2016) 042310.

[15] Kondakci H. E., Abouraddy A. F. and Saleh B. E., Nat. Phys., 11 (2015) 930.

[16] Kondakci H. E., Abouraddy A. F. and Saleh B. E., Optica, 2 (2015) 201.

[17] Kondakci H. E., Szameit A., Abouraddy A. F., Christodoulides D. N. and Saleh B. E. A., Optica, 3 (2016) 477.

[18] Kondakei A. E., Keil R., Szameit A., Abouraddy A. F., Christodoulides D. N. and Saleh B. A., Tailoring photon-number distribution in disordered lattices with chiral symmetry presented at 2016 CLEO (Lasers and Electro-Optics) Conference (IEEE) 2016.

[19] Kondakci H. E., Abouraddy A. F. and Saleh B. E. A., Sci. Rep., 7 (2017) 8948.

[20] Szameit A., Nat. Phys., 11 (2015) 895.

[21] Makris K. G., El-Ganainy R., Christodoulides D. N. and Musslimani Z. H., Phys. Rev. Lett., 100 (2008) 103904.

[22] Miri M.-A., Aceves A. B., Kottos T., Kovanis V. and Christodoulides D. N., Phys. Rev. A, 86 (2012) 033801.

[23] Luo X., Huang J., Zhong H., Qin X., Xie Q., Kivshar Y. S. and Lee C., Phys. Rev. Lett., 110 (2013) 243902.

[24] Kondakci H. E., Martin L., Keil R., Perez-Leija A., Szameit A., Abouraddy A. F., Christodoulides D. N. and Saleh B. E. A., Phys. Rev. A, 94 (2016) 021804 . 